\title{
Can Effects of Dark Matter Be Explained by the Turbulent Flow of Spacetime?
}

\author{
F. Elliott Koch, Angus H. Wright \\ School of Physics, University of New South Wales, Sydney, Australia \\ Email: f.koch@unsw.edu.au,mhchan@phy.cuhk.edu.hk
}

Received July 9, 2012; revised August 11, 2012; accepted August 18, 2012

\begin{abstract}
For the past forty years the search for dark matter has been one of the primary foci of astrophysics, although there has yet to be any direct evidence for its existence [1]. Indirect evidence for the existence of dark matter is largely rooted in the rotational speeds of stars within their host galaxies, where, instead of having a $\sim r^{-1 / 2}$ radial dependence, stars appear to have orbital speeds independent of their distance from the galactic center, which led to proposed existence of dark matter $[1,2]$. We propose an alternate explanation for the observed stellar motions within galaxies, combining the standard treatment of a fluid-like spacetime with the possibility of a "bulk flow" of mass through the Universe. The differential "flow" of spacetime could generate vorticies capable of providing the "perceived" rotational speeds in excess of those predicted by Newtonian mechanics. Although a more detailed analysis of our theory is forthcoming, we find a crude "order of magnitude" calculation can explain this phenomena. We also find that this can be used to explain the graviational lensing observed around globular clusters like "Bullet Cluster".
\end{abstract}

Keywords: Dark Matter; Galaxy; Relativity; Gravitation

\section{Introduction}

In the pursuit of determining a model that accurately predicts the past, present, and future of the evolution of the Universe, physicists have generated a range of possible candidates. Currently, the most generally accepted being the $\Lambda$ CDM model, which contains, among others, parameters dealing with the existence of a cosmological constant $(\Lambda)$ and cold dark matter (CDM). Furthermore, the existence of the dark matter component of this cosmology, and others, is not that which is generally considered contentious. Dark matter has rather become somewhat of a staple in the diet of cosmologies. However, there are observational reasons to give pause to the assumed existence of Universal cold dark matter, which then should lead us to question whether or not there are other alternative models.

Models of dark matter succeed in accounting for the galactic rotation curves observed throughout the Universe, by increasing the mass of the galaxy beyond the observed. There are, however, simple problems with the dark matter halo model that have yet to be fully explained (e.g. [3-7]). One of these problems is the disparity between the observed (stellar) mass function, usually defined in terms of the Schechter function, and the theoretically expected cosmological halo mass function [8]. One of the defining problems of galactic formation and evolution is determining the origins of this disparity. This is an example of how our understanding of dark matter (or lack there of) is still grounds for much debate. However, if it may be possible to utilise a different model for the origin of galactic formation then it is possible that some of these questions may be answered.

Recently, there has been some observational evidence for the "bulk flow" of matter through the Universe [9-13]. If these measurements prove to be true, then the nature of this flow is of interest beyond that of the distribution of matter in the Universe. Specifically relevant to this discussion is the interpretation that the "flow" observed is caused not by an en masse transit of matter through the Universe, but rather by the motion of spacetime itself. Whilst this concept is indeed foreign it can be considered somewhat preferential to the former case, from an isotropic viewpoint, as the motions of objects in the Universe need not be preferentially oriented in this regime. More importantly, variation in the "bulk flow" of spacetime fluid through the Universe could produce eddies in spacetime and provide the additional unexplained velocity to rotational speeds of stars beyond the central bulge of galaxies.

As the intention of this paper is to merely propose an alternative theoretical explanation for observations consistent with the existence of dark matter the structure is 
as follows: Section 2 describes the treatment of spacetime as a fluid. Sections 3 and 4 discuss classical fluid dynamics and relativistic fluid dynamics and how votices in the spacetime "fluid" can produce observations consisten with dark matter. Finally we present concluding remarks and propose future work in Section 5.

\section{Spacetime as a Fluid and the Differential Rotation of Spacetime}

In General Relativity it is common for theories to compare the nature of the spacetime coordinate system to an ideal fluid, often called the "cosmic fluid". This treatment is integral for the formulation of many concepts in GR, including the formation and propagation of gravitational waves. Additionally, Kerr-Newman geometry for a rotating black hole, which predicts an effective "differencial rotation" of spacetime. The principle effect regarding black hole studies is the implication that, within $r_{0} \equiv M+\sqrt{M^{2}-a^{2} \cos ^{2} \theta}$, it is impossible to remain stationary with respect to distant "stationary" observer beyond $r_{0}$.

The Kerr-Newman geometery has been used to successfully describe the light curves of rapidly rotating neutron stars [14]. Doppler boosting and time-delayinduced pertubations from frame-dragging cause "soft lags" in pulse profiles of neutron stars which have been measured in X-ray spectra.

As a starting point for our proposition we use this geometry, simply as an example of how the differential rotation of spacetime is possible. We leave all other blackhole allegories or implications of a large, dense, rotating mass located at the "centre of the Universe" behind.

\section{Vortices in Classical Fluid Dynamics}

If spacetime is able to experience a form of differential rotation, then it is of interest to examine how this would impact the fluid treatment we noted earlier.

Classical fluid dynamics states that eddies/vortices with angular velocity $\omega^{\text {vort }}$ occur for any differential flow as:

$$
\omega^{\text {vort }}=\nabla \times \mathbf{v}
$$

and

$$
\frac{\partial \omega^{\text {vort }}}{\partial t}=\nabla \times\left(\omega^{\text {vort }} \times \mathbf{v}\right) .
$$

Due to the chaotic nature of this relationship, when applied to astronomical distances and timescales even small perturbations can ultimately produce large scale phenomena.

Essentially, if we treat spacetime as a classical fluid with turbulence caused by a differential flow as observed by Osborne et al. [12] (and others), spatial variations in the flow would generate eddies that could not only host galaxies, but adequately explain their rotational dynamics as well.

Treating spacetime as a classical fluid, and assuming the velocity of any "bulk flow" is

$\mathbf{v}=v_{x}(x, y) \hat{x}+v_{y}(x, y) \hat{y}$,

we find $\omega_{z}^{\text {vort }}=\left(\delta v_{x}^{2}+\delta v_{y}^{2}\right)^{1 / 2}$ where $\delta v_{i}=\partial v_{i} / \partial x_{j \neq i}$. van Albada et al. [15] utilised light curves to show that rotational velocities of stars deviated from Newtonian mechanics by $\sim 50 \mathrm{~km} / \mathrm{s}$ at a radial distance of $\sim 20$ $\mathrm{kpc}$ from the galactic centre. We find that, to account for this discrepancy $\Delta v \equiv\left(\delta v_{x}^{2}+\delta v_{y}^{2}\right)^{1 / 2}$,

$\omega^{\text {vort }}=\Delta v \sim 3 \mathrm{kms}^{-1} \mathrm{kpc}^{-1}$ for observed velocities of the outer most stars of a galaxy which correspond to the largest values of $\Delta v$. So, given some bulk flow through in the local Universe, there only need be a systematic variation of $\sim 3 \mathrm{kms}^{-1} \mathrm{kpc}^{-1}$ within the flow to produce an eddy large enough to provide the unaccounted velocity to the outermost stars of a galaxy. Furthermore, as the velocity of the eddy is dependent on radius from the centre of the eddy (i.e. the galactic centre), the effect will diminish as the radius from the galactic centre decreases. Accounting for the increase in observable galactic mass with decreasing radius, it can be supposed that as one approaches the galactic centre the motion of stars becomes primarily governed by gravitation. Formulated simply: as $r \rightarrow r_{c}, v_{*} \rightarrow v_{N}$, where $r_{c}$ is the radius of the galactic "bulge" and $v_{N}$ is the orbital speed of stars predicted by Newtonian mechanics.

In this way, we propose that the observed motion of galactic stars over the entire disk may be explained by the presence of eddies in spacetime caused by appreciably small variations in "bulk flow".

Thus far we have treated spacetime as a classical fluid, but as we are dealing with distortions of spacetime relativistic effects must be addressed. Greenberg found that, independent of any relativistic geometry used, the angular velocity 2 -form of an eddy/vortex is

$$
\omega_{\alpha \beta}=\frac{1}{2}\left(u_{\alpha ; \beta}-u_{\beta ; \alpha}\right)
$$

where $u_{\beta}$ is the velocity 4 -vector of the "fluid" [16]. The most noticeable difference between Equations (1) and (2) is the coupled nature of spacetime. Therefore any changes in the "flow" over time could also produce vortices in spacetime, much like those produced by variations in differential flow in classical dynamics. Coupling temporal and spatial variations could further enhance the turbulent nature of spacetime, and thus the production of eddies where galaxies could grow.

\section{Relativis Fluid Dynamics}

Examining the relativistic case, we can choose a refer- 
ence frame such that $u_{t} \neq 0$, and the spatial components $u_{i}$ are all zero. The reasoning behind this is that, for an external observer, the motion of a "fluid" through a stationary reference frame is observationally indifferent to a static "fluid" in a co-moving reference frame. Using this restriction, Equation (2) becomes

$$
\omega_{t i}=-\omega_{i t}=\frac{1}{2} u_{t ; i}
$$

Since this essentially a 2-form for a vortex in spacetime, the magnitude of the resulting rotation would be

$$
\omega^{2}=\omega_{\alpha \beta} \omega^{\alpha \beta}=\omega_{\alpha \beta} g^{\alpha \gamma} g^{\beta \delta} \omega_{\gamma \delta},
$$

which is the magnitude of the rotation for the vortex, squared. Since it contains products of $\omega_{\mu \nu} \omega_{\nu \mu}$, then, in principle, $\omega^{2}$ can be non-zero. Additionally, the covariant derivative embedded in $\omega_{\mu \nu}$ provides us with information on variations within $g_{\mu v}$ (spacetime) that yield $\omega^{2} \neq 0$, possibly explaining observed phenomena.

We reserve the comprehensive mathematical analysis for a forthcoming paper and merely present a simplified "glimpse" of the interpretation of Equation (4). If a vortex in spacetime is to be used as a possible explanation for the observed rotational velocities of stars, and on average, these velocities are independent of angular position within the galaxy as well as vertical position within the disc, we have assumed that $u_{t ; \phi}=u_{t ; z}=0$, hence

$$
\begin{aligned}
\omega^{2} & =2 \omega_{r t}^{2}\left[g^{t t} g^{r r}-\left(g^{t r}\right)^{2}\right] \\
& =\frac{1}{4}\left(\partial_{r} u_{t}-\Gamma_{r t}^{t} u_{t}\right)^{2}\left[g^{t t} g^{r r}-\left(g^{t r}\right)^{2}\right],
\end{aligned}
$$

because $g^{t r}=g^{r t}$ and $\omega_{t r}=-\omega_{r t}$. This can be used as a restriction for properties of $g_{\mu v}$ and expand upon existing geometries such as the Kerr-Newman and/or Friedmann-Robertson-Walker metrics, which will be presented in subsequent publications. Here we merely present evidence that small perturbations in $g_{\mu v}$ and $u_{t}$ may be used to explain the rotational speeds of stars in other galaxies.

Assuming the metric for the local spacetime is essentially flat, $g_{\mu v}=\delta_{\mu v}+\delta g_{\mu v}$, as well as the variations in $u_{t} \delta u_{t} \equiv \partial_{r} u_{t}$ and the variations in $g$ as

$\delta g u_{t} \equiv \Gamma_{r t}^{t} u_{t}$, we can approximate the magnitudes of these "variations" that could explain observed phenomena. Since we are assuming cross terms are small and $g^{t t} \sim g^{r r} \sim 1, \omega^{2} \sim\left(\delta u_{t}-\delta g u_{t}\right)^{2}$. In the extreme case where the rotational speed of stars for a galaxy, as predicted by theory is zero, $v_{*}=\omega r_{\text {? }}$ or

$\omega=v_{*} / r_{?} \sim 10^{-4} \mathrm{kms}^{-1} \mathrm{Lyr}^{-1}$ and that $\delta u_{t}=0$ implying that the stellar motion is purely from variations in the geometry. Coupling this with the afore mentioned indifference of motion through spacetime and spacetime moving with the "fluid" we can approximate the magnitude of $\delta g$. Based on observations of Osborne et al. [12], we assume that $u_{t} \sim 100 \mathrm{kms}^{-1}$. Therefore, we determine that $\delta g \sim 10^{-6} \mathrm{Lyr}^{-1}$ could produce effects consistent with stellar rotational velocities observed. Additionally, Osborne et al. [12] also found that the "flow" changed by $\sim 50 \mathrm{kms}^{-1}$ between the redshifts of 0.4 and 0.8 . Again, if this is largely due to variations in the geometry of spacetime, this would imply $\delta g \sim 10^{-8} \mathrm{kms}^{-1}$. Though this is smaller than the previous approximation, that approximation did not account for the motion of stars from Newtonian mechanics.

\section{Summary}

If the observed effects attributed to dark matter are indeed caused by the turbulent flow of spacetime, then we can simply hypothesise that any galaxy in a cluster formed in this way should all rotate in the same direction. Albeit only significant to $1.6 \sigma$, evidence for this effect has been detected by Longo et al. [17]. There is no reason to expect that this observation would also be caused in the dark matter Regime for reasons other than chance. As this is a simple method to determine if turbulent spacetime flows may be the cause of galactic rotational curves, Doppler measurements of stellar velocities in galaxies are extremely important. Furthermore, if rotational curves for distant galaxies can be found, isotropy measurements could serve as an additional constraint for the validity of this theory.

Finally, we find that chaotic flows could exist on both "large" and "small" scales. Large scale turbulence is dominated by the "flow" velocity and the uniformity thereof. In this context, "large" scale turbulence would be on a galactic scale, with "large" eddies being comparable to the size of a galaxy, which could be used to explain why galaxies are not "sheared" apart. Small scale turbulence is dominated by the viscosity of the flow, which would most likely be caused by the gravitational attraction of masses present in the region of the eddy, as mentioned previously in relation to the galactic rotation curves. The scale of a "small eddy" would be comparable to that of stellar clusters. Since these clusters are still affected by distortions in spacetime, this could explain the observed gravitational lensing caused by some stellar clusters that could not be explained by modified Newtonian dynamics (MOND) [18].

This concept can, therefore, effectively explain the major observations that lead to the introduction of dark matter, and removes the need for the existence of a massive dark matter halo about galaxies. If this theory is correct, galaxies co-located within a differentially moving frame, should all rotate in the same direction (i.e. same chirality). Additionally, when observed from an external reference frame, there should be variations in the 
local spacetime of a galaxy $\delta g u_{t} \sim 10^{-4} \mathrm{kms}^{-1} \mathrm{Lyr}^{-1}$ where $u_{t}$ is the observed "flow" of the surrounding galaxies.

Finally can now draw some different, interesting, conclusions from the disparity between the observed stellar mass function and the cosmological halo mass function. Using the model we propose here, the origins of galactic evolution lie not in vast halos of dark matter, but rather in the turbulence of spacetime. The turbulence itself traces back its origins to spatial and temporal variations in the motion of spacetime. If we are able to conceptualise this method of seeding galaxies, then we can also recognise that the formation of the vortices in which galaxies originate is highly dependent on the mechanics of the local spacetime. This, when interpreted simply, means that the expected distribution of galactic mass (i.e. the observed stellar mass function) should not be conformal to a simplistic power law (i.e. the cosmological halo mass function), but rather should be more complex in nature. The true distribution of vortex sizes in a field of uniform variation should be expected to be inately coupled with galactic mass. This may well explain the shape of the Schechter function, and why galactic mass function does not follow a simple power law, without the need for complex models to explain the reduction of star formation in the low and high mass dark matter halo regimes.

As stated above, many of these conclusion are too complicated to present in any detail within this paper, of which will be presented in following papers. The purpose of this paper is to merely propose an alternate explanation for the observed effects of dark matter and a possible explanation for why dark matter is not consistent with some other theories and observation.

\section{Acknowledgements}

The authors would like to thank Graeme Salter, Jonathan Horner, Annant Tanna and Bradley Hansen for insightful discussions regarding the formulation of this theory. FEK would also like to thank UNSW and the ARC for financial support used during the development of this theory under grants PS27228-FRGP, PS27261-ECR and DP120105045.

\section{REFERENCES}

[1] T. A. Porter, R. P. Johnson and P. W. Graham, "Dark Matter Searches with Astroparticle Data,” Annual Review of Astronomy and Astrophysics, Vol. 49, 2011, pp. 155194. doi:10.1146/annurev-astro-081710-102528

[2] P. J. E. Peebles, "Principles of Physical Cosmology," 1993.

[3] A. Klypin, A. V. Kravtsov, O. Valenzuela and F. Prada, "Where Are the Missing Galactic Satellites?” Astro- physical Journal, Vol. 522, No. 1, 1999, pp. 82-92. doi:10.1086/307643

[4] S. Garbari, J. I. Read and G. Lake, "Limits on the Local Dark Matter Density," Monthly Notices of the Royal Astronomical Society, Vol. 416, No. 3, 2011, pp. 2318-2340. doi:10.1111/j.1365-2966.2011.19206.x

[5] I. D. Karachentsev, "Missing Dark Matter in the Local Universe,” Astrophysical Bulletin, Vol. 67, No. 2, 2012, pp. 123-134. doi:10.1134/S1990341312020010

[6] V. Poitras, "Constraints on _(t)-Cosmology with Power Law Interacting Dark Sectors,” Journal of Cosmology and Astroparticle Physics, Vol. 6, 2012, p. 39. doi:10.1088/1475-7516/2012/06/039

[7] D. Sluse, V. Chantry, P. Magain, F. Courbin and G. Meylan, "COSMOGRAIL: The Cosmological Monitoring of Gravitational Lenses. X. Modeling Based on HighPrecision Astrometry of a Sample of 25 Lensed Quasars: Consequences for Ellipticity, Shear, and Astrometric Anomalies,” A \& A, Vol. 538, 2012, p. A99.

[8] B. P. Moster, R. S. Somerville, C. Maulbetsch, F. C. van den Bosch, A. V. Macci`o, T. Naab and L. Oser, "Constraints on the Relationship between Stellar Mass and Halo Mass at Low and High Redshift,” Astrophysical Journal, Vol. 710, No. 2, 2010, pp. 903-923. doi:10.1088/0004-637X/710/2/903

[9] B. A. Benson, S. E. Church, P. A. R. Ade, J. J. Bock, K. M. Ganga, J. R. Hinderks, P. D. Mauskopf, B. Philhour, M. C. Runyan and K. L. Thompson, "Peculiar Velocity Limits from Measurements of the Spectrum of the Sunyaev Zeldovich Effect in Six Clusters of Galaxies,” Astrophysical Journal, Vol. 592, 2003, pp. 674-691.

[10] S. Bhattacharya and A. Kosowsky, "Dark Energy Constraints from Galaxy Cluster Peculiar Velocities,” Physical Review D, Vol. 77, 2008, Article ID: 083004. doi:10.1103/PhysRevD.77.083004

[11] H. A. Feldman, R. Watkins and M. J. Hudson, "Cosmic Flows on 100 h-1 Mpc Scales: Standardized Minimum Variance Bulk Flow, Shear and Octupole Moments," Monthly Notices of the Royal Astronomical Society, Vol. 407, No. 4, 2010, pp. 2328-2338. doi:10.1111/j.1365-2966.2010.17052.X

[12] S. J. Osborne, D. S. Y. Mak, S. E. Church and E. Pierpaoli, "Measuring the Galaxy Cluster Bulk Flow from WMAP Data," Astrophysical Journal, Vol. 737, No. 2, 2011, p. 98. doi:10.1088/0004-637X/737/2/98

[13] S. J. Turnbull, M. J. Hudson, H. A. Feldman, M. Hicken, R. P. Kirshner and R. Watkins, "Cosmic Flows in the nearby Universe from Type Ia Supernovae,” Monthly Notices of the Royal Astronomical Society, Vol. 420, No. 1, 2012, pp. 447-454. doi:10.1111/j.1365-2966.2011.20050.x

[14] T. M. Braje, R. W. Romani and K. P. Rauch, "Light Curves of Rapidly Rotating Neutron Stars,” Astrophysical Journal, Vol. 531, No. 1, 2000, pp. 447-452. doi:10.1086/308448

[15] T. S. van Albada and R. Sancisi, "Dark Matter in Spiral Galaxies,” Royal Society of London Philosophical Transactions Series A, Vol. 320, No. 1556, 1986, pp. 447-464. doi:10.1098/rsta.1986.0128 
[16] C. W. Misner, K. S. Thorne and J. A. Wheeler, "Gravitation,” W.H. Freeman and Co., San Francisco, 1973.

[17] M. J. Longo, "Detection of a Dipole in the Handedness of Spiral Galaxies with Redshifts z 0.04,” Physics Letters $B$, Vol. 699, No. 4, 2011, pp. 224-229.

doi:10.1016/j.physletb.2011.04.008
[18] R. Ibata, A. Sollima, C. Nipoti, M. Bellazzini, S. C. Chapman and E. Dalessandro, "The Globular Cluster NGC 2419: A Crucible for Theories of Gravity,” Astrophysical Journal, Vol. 738, No. 2, 2011, p. 186. doi:10.1088/0004-637X/738/2/186 